BULL. AUSTRAL. MATH. SOC.

VOL. $33(1986), 307-318$

\title{
WEIGHTED FUNCTION ALGEBRAS ON GROUPS AND SEMIGROUPS
}

\author{
HENERI A.M. DZinOTYIWEYI
}

\begin{abstract}
For a locally compact topological group admitting a weight function, we establish necessary and sufficient criteria for all the weighted continuous functions to be weakly almost periodic. Among other results, we show that weak almost periodicity of all $\dot{\omega}$-weighted continuous functions on a discrete semigroup $S$, can be very different drom the phenomenon of regularity of multiplication in the weighted algebra $\ell^{l}(S, \omega)$.
\end{abstract}

\section{Introduction}

Let $S$ be a locally compact topological semigroup, $\omega$ a continuous weight function on $S$ and $C(S, \omega)$ the space of all continuous $\omega$-weighted functions on $S$. When $S$ is a group (and $\omega=1$ ), it is well known that all functions in $C(S, 1)$ are weakly almost periodic if and only if $S$ is compact - see for example [1] or [3]. In this paper we establish necessary and sufficient criteria for all functions in $C(S, \omega)$ to be weakly almost periodic for the case where $S$ is a group or a cancellative discrete semigroup.

When $S$ is discrete, it is known that all functions in $C(S, 1)$ are weakly almost periodic if and only if the algebra $\ell^{l}(S)$ has regular multiplication - see for example [8] or [3, Theorem 4.5.5(ii)]. One may ask whether a similar result holds for the corresponding weighted algebras. We show that the situation for weighted algebras can be very different

Among other results, we also prove that, if $S$ is a group, the

Received 19 July 1985. Paper presented at the Third Symposium of the Southern Africa Mathematical Sciences Association, University of Zambia, December 1984 .

Copyright Clearance Centre, Inc. Serial-fee code: 0004-9727/86 $\$ A 2.00+0.00$. 
weighted group algebra $L^{1}(G, \omega)$ contains a weakly compact element if and only if $G$ is compact. This improves earlier results of Ghahramani ([5] and [6]).

In section 2 we introduce our notation and prove some elementary properties of a weight function. Our main results are given in section 3 .

2. Notation and some elementary properties of weight functions.

Throughout this paper, let $S$ denote a topological semigroup. A positive function $\omega$ defined on $S$ is called a weight function if

$$
\omega(x y) \leqslant \omega(x) \omega(y) \text { for all } x, y \in S .
$$

It is interesting to note that measurable weight functions on locally compact groups are bounded away from both zero and infinity on compact sets. This is what our next result says.

PROPOSITION 2.1. Let $K$ be a compact subset of a locally compact giroup $G$ and $\omega$ a measurable weight function on $G$. Then there exists $a, b \in \mathbb{R}$ such that

$$
0<a \leqslant \omega(x) \leqslant b \quad \text { for all } x \in K
$$

Proof. First we establish the existence of $b$. To this end, let $\lambda$ be a Haar measure on $G$ and set $U_{n}:=\{x \in G: \omega(x)<n\}$. Clearly $\infty$ $\bigcup_{n=1} U_{n}=G$ and each $U_{n}$ is measurable. Hence $\lambda\left(U_{m}\right)>0$ for some $n=m$. The interior of $U_{m}^{2}$ is non-empty - see for example ([3], pp. 17 and 18). Let $z \in \operatorname{int}\left(U_{m}{ }^{2}\right)$ and set $V:=\operatorname{int}\left(z^{-1} U_{m}{ }^{2}\right)$, and note that $V$ is an open neighbourhood of the identity. By the compactness of $K$ we can find a finite number of points $y_{1}, \ldots, y_{t}$ in $K \cup K^{-1}$ such that

$$
K \cup K^{-1} \subset V y_{1} \cup \ldots \cup v y_{t} .
$$

Let $b:=m^{2} \omega\left(z^{-1}\right) \cdot \max \left\{\omega\left(y_{i}\right): 1 \leqslant i \leqslant t\right\}$. Then if $x \in K \cup K^{-1}$ we have $x=v y_{i}$ for some $v \in V$ and $i \in\{1, \ldots, t\}$, and so

$$
\omega(x)=\omega\left(v y_{i}\right) \leqslant \omega(v) \omega\left(y_{i}\right) \leqslant m^{2} \omega\left(z^{-1}\right) \omega\left(y_{i}\right) \leqslant b .
$$

Thus $\omega(x) \leqslant b$, for all $x \in K \cup K^{-1}$. 
Next let $a:=\inf \{\omega(x): x \in K\}$ and suppose $a=0$. Then there exists a sequence $\left\{x_{n}\right\}$ in $K$ such that $\omega\left(x_{n}\right) \rightarrow 0$. Since $1 \leqslant \omega\left(x_{n}\right) \omega\left(x_{n}^{-1}\right)$, we must have $\omega\left(x_{n}^{-1}\right) \rightarrow \infty$, which contradicts $(*)$. Hence we must have $a>0$.

REMARK. Of course there are many measurable weight functions that are not continuous. For example on the additive group $\mathbb{R}$ with the usual topology one may consider the weight function $\omega$ given by

$$
\omega(x):= \begin{cases}1 & \text { if } x \in[0,1] \\ 2 & \text { elsewhere. }\end{cases}
$$

PROPOSITION 2.2. Let $\omega$ be a weight function on $S$ such that $\{x \in S: \omega(x)<\varepsilon\}$ is finite for some $\varepsilon>0$. Then $\omega(x) \geqslant 1$, for alz $x \in S$.

Proof. Suppose $\omega(x)<1$ for some $x \in S$. Choose $p \in N$ such that $\omega\left(x^{p}\right)<\varepsilon$. Then

$$
\omega\left(x^{p+n+1}\right) \leqslant \omega\left(x^{p+n}\right) \omega(x)<\omega\left(x^{p+n}\right) \leqslant \omega\left(x^{p}\right) \omega(x)^{n}<\varepsilon .
$$

Thus $\left\{x^{p+n}: n \in N\right\}$ is an infinite subset of $\{y \in S: \omega(y)<\varepsilon\}-$ contrary to the hypothesis. Thus the result follows.

COROLLARY 2.3. If $\omega$ is a measurable weight function on a compact topological group $G$, then $\omega(x) \geqslant 1$ for all $x \in G$.

Proof. This follows trivially from Propositions 2.1 and 2.2.

2.4. Let $\mathbb{C}(S)$ be the set of all complex-valued continuous functions on $S, C(S)$ the space of all bounded functions in $\Phi(S)$ under the supremum norm \|\|$_{S}$ and $\omega$ a continuous weight function on $S$. We define the space of weighted continuous functions $C(S, \omega)$ by

$$
C(S, \omega):=\{f \in \Phi(S): f \omega \in C(S)\}
$$

with the norm given by $\|f\|_{\omega}:=\|f \omega\|_{S}$.

Let

$$
\begin{array}{r}
W A P(S, \omega):=\left\{f \in C(S, \omega):\left\{{ }_{x} f: x \in S\right\}\right. \text { is a relatively weakly } \\
\text { compact subset of } C(S, \omega)\}
\end{array}
$$

and 


$$
\begin{array}{r}
A P(S, \omega):=\left\{f \in C(S, \omega):\left\{_{x} f: x \in S\right\}\right. \\
\text { compact subset of } C(S, \omega)\},
\end{array}
$$

where

$$
x_{x} f(y):=f(x y) \text { for all } x, y \in S \text {. }
$$

When $\omega=1$ we get the familiar spaces $\operatorname{WAP}(S)=\operatorname{WAP}(S, 1)$, $A P(S)=A P(S, 1)$ and $C(S)=C(S, 1)$.

PROPOSITION 2.5. A function $f$ belongs to $W A P(S, \omega)$ if and only if $\left.{ }_{{ }_{x}} f \cdot \omega: x \in S\right\}$ is a relatively weakly compact subset of $C(S)$.

Proof. A standard argument (which we omit) shows that the map $f \rightarrow f . \omega$ of $C(S, \omega)$ into $C(S)$ is weakly a topological homomorphism and hence the result follows.

2.6. For a discrete semigroup $S$ with weight function $\omega$, the set $\ell^{1}(S, \omega)$ of all complex-valued functions $f$ on $S$ such that $f . w \in \ell^{1}(S)$, is a Banach algebra with convolution as multiplication and norm given by

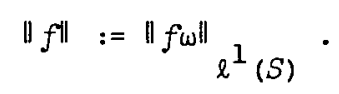

If $\omega$ is a measurable weight function on a locally compact topological group $G$, we have the weighted convolution algebra $L^{1}(G, \omega)$ of (equivalence classes of) all measurable complex-valued functions $f$ on $G$ such that $f . \omega \in L^{1}(G)$ under the norm $\|f\|:=\|f \cdot \omega\| L_{L}^{1}(G)$.

2.7. The reader can easily note that our definitions for the spaces $W A P(S, \omega)$ and $A P(S, \omega)$ contain the requirement that the functions $f \in C(S, \omega)$ involved be such that $x^{f \in C(S, \omega)}$ for all $x \in S$. This is related to the finiteness of $\Omega(x)$ where

$$
\Omega(x):=\sup \left\{\frac{\omega(y)}{\omega(x y)}: y \in S\right\}
$$

for all $x \in S$ - see for example Lemma 3.1.

\section{The main results}

LEMMA 3.1. Let $\omega$ be a continuous weight function on $S$. Then ${ }_{x} f \in C(S, \omega)$, for all $f \in C(S, \omega)$ and $x \in S$, if and only if $\Omega(x)$ 
is finite.

Proof. To establish the necessary condition, we note that

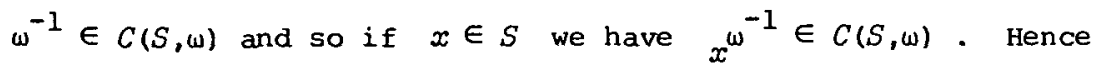

$$
\infty>\|_{x^{\omega}}{ }^{-1_{\|}}=\sup \left\{\frac{\omega(y)}{\omega(x y)}: y \in S\right\}=\Omega(x) \text {. }
$$

Conversely, suppose $\Omega(x)$ is finite, for all $x \in S$, and let $f \in C(S, \omega)$. Thus, for all $x, y \in S$, we have that

$$
|f(y) \omega(y)|=|f(x y) \omega(y)|=|f(x y) \omega(x y)| \frac{\omega(y)}{\omega(x y)} \leqslant \| f_{\omega} \Omega(x)
$$

and so

$$
\|\|^{f \|_{\omega}<\infty} \text { or } x^{f \in C(S, \omega)} \text {. }
$$

COROLLARY 3.2. If $\omega$ is a continuous weight function on a locally

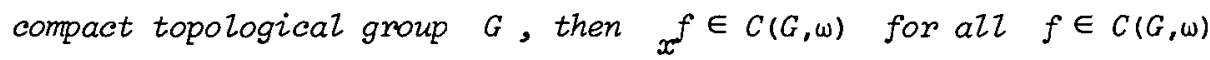
and $x \in G$.

Proof. For all $x$ and $y$ in $G$, we have

$$
\frac{\omega(y)}{\omega(x y)}=\frac{\omega\left(x^{-1} x y\right)}{\omega(x y)} \leqslant \frac{\omega\left(x^{-1}\right) \omega(x y)}{\omega(x y)}=\omega\left(x^{-1}\right)
$$

and so $\Omega(x) \leqslant \omega\left(x^{-1}\right)$. By 3.1 our result follows.

The reader is asked to bear the preceding result in mind throughout tine proof of our next theorem.

THEOREM 3.3. Let $\omega$ be a continuous weight function on a locally compact topological group $G$. Then, the following are equivalent

(i) $\operatorname{WAP}(G, \omega)=C(G, \omega)$;

(ii) either (a) $G$ is compact

or (b) $G$ is a countable discrete group such that

$$
\begin{aligned}
& \lim \lim \frac{\omega\left(y_{m}\right)}{\omega\left(x_{n} y_{m}\right)}=0 \text {, whenever }\left\{x_{n}\right\} \text { and }\left\{y_{m}\right\} \\
& \text { are sequences of distinct points of } G \text {. }
\end{aligned}
$$

Proof. Suppose (i) holds and set $V_{k}:=\{x \in G: \omega(x) \leqslant k\}$. We claim that either $V_{k}$ is finite $(k \in \mathbb{N})$ or $G$ is compact. To establish our claim, suppose for some $k$ (now fixed) $V_{k}$ is infinite and 
$G$ is not compact. Then, by an argument similar but simpler to that we employed in ([4], Theorem 7.3), there exist sequences $\left\{x_{n}\right\} \subset V_{k}$ and $\left\{y_{m}\right\} \subset G$, and a positive function $h$ in $C(G)$ such that

$$
h\left(x_{n} y_{m}\right)=\left\{\begin{array}{lll}
0 & \text { if } & n<m \\
1 & \text { if } & n \geqslant m
\end{array}\right.
$$

Then $f:=h \omega^{-1}$ is a function in $C(G, \omega)$ and

(*) $\quad x_{n} f\left(y_{m}\right) \omega\left(y_{m}\right)=f\left(x_{n} y_{m}\right) \omega\left(y_{m}\right)=\frac{h\left(x_{n} y_{m}\right) \omega\left(y_{m}\right)}{\omega\left(x_{n} y_{m}\right)}= \begin{cases}0 & \text { if } n<m \\ C(n, m) & \text { if } n \geqslant m ;\end{cases}$ where

$$
C(n, m):=\frac{\omega\left(y_{m}\right)}{\omega\left(x_{n} y_{m}\right)} \geqslant \frac{\omega\left(y_{m}\right)}{\omega\left(x_{n}\right) \omega\left(y_{m}\right)}=\frac{1}{\omega\left(x_{n}\right)} \geqslant \frac{1}{k} .
$$

So, by Grothendieck ([7], Theorem 6), equation (*) implies that $\left\{_{x} f . \omega: x \in G\right\}$ is not relatively weakly compact. Recalling

Proposition 2.5 we have $f \in C(G, \omega) \backslash W A P(G, \omega)$, which contradicts (i). Hence each $V_{k}$ is finite or $G$ is compact.

Next suppose $G$ is not compact and choose $p \in N$ such that $w(1)<p$. Then $V_{p}$ is a finite neighbourhood of the identity, so $G$ is discrete: As $G=\bigcup_{k=1}^{\infty} V_{k}$ we also have $G$ countable. Since each $V_{k}$ is finite, if $\left\{z_{n}\right\}$ a sequence of distinct points of $G$, then

$$
\omega\left(z_{n}\right) \rightarrow \infty \text { as } n \rightarrow \infty
$$

Now let $\left\{x_{n}\right\}$ and $\left\{y_{n}\right\}$ be any sequences of distinct points in $G$. The function $f:=\omega^{-1}$ is in $C(G, \omega)$ and hence in $\operatorname{WAP}(G, \omega)$. Let

$$
d:=\varlimsup_{n} \underset{m}{\lim } f\left(x_{n} y_{m}\right) \omega\left(y_{m}\right)=\overline{\lim }_{n} \varlimsup_{m} \frac{\omega\left(y_{m}\right)}{\omega\left(x_{n} y_{m}\right)}
$$

(where $d$ may be infinite). From (1), we get

$$
\lim _{m} \lim _{n} f\left(y_{m}\right) \omega\left(y_{m}\right)=\underset{m}{\lim } \lim _{n} \frac{\omega\left(y_{m}\right)}{\omega\left(x_{n} y_{m}\right)}=\underset{m}{\lim 0}=0
$$


Since $\left\{{ }_{x} f \cdot \omega: x \in G\right\}$ is relatively weakly compact (by (i) and Proposition 2.5), Grothendieck (171, Theorem 6) and (2) imply that $d=0$ Consequently

$$
\lim _{n} \lim _{m} \frac{\omega\left(y_{m}\right)}{\omega\left(x_{n} y_{m}\right)}=0
$$

Thus (i) implies (ii).

Now suppose (ii) (a) holds. Then

$$
W A P(G)=C(G)=C(G, \omega)
$$

and the map $f \rightarrow f . \omega$ of $C(G)$ into $C(G, \omega)$ is weakly continuous. Hence $\{f f \omega: x \in G\}$ is a relatively weakly compact subset of $C(G, \omega)$, for all $f \in C(G, \omega)$. Thus $\operatorname{WAP}(G, \omega)=C(G, \omega)$, by Proposition 2.5.

Next suppose (ii) (b) holds. Let $f$ be any non-negative function in $C(G, \omega)$ and set $M:=\|f\|_{\omega}$, Then if $\left\{x_{n}\right\}$ and $\left\{y_{m}\right\}$ are any sequences, as specified in (ii) (b), we have

$$
0 \leqslant x_{n} f\left(y_{m}\right) \omega\left(y_{m}\right)=f\left(x_{n} y_{m}\right) \omega\left(x_{n} y_{m}\right) \frac{\omega\left(y_{m}\right)}{\omega\left(x_{n} y_{m}\right)} \leqslant M \frac{\omega\left(y_{m}\right)}{\omega\left(x_{n} y_{m}\right)}
$$

and

$$
0<\frac{1}{\omega\left(x_{n}\right)}=\frac{\omega\left(x_{n} y_{m}\right)}{\omega\left(x_{n}\right) \omega\left(x_{n} y_{m}\right)} \leqslant \frac{\omega\left(x_{n}\right) \omega\left(y_{m}\right)}{\omega\left(x_{n}\right) \omega\left(x_{n} y_{m}\right)}=\frac{\omega\left(y_{m}\right)}{\omega\left(x_{n} y_{m}\right)}
$$

Hence

$$
\lim _{n} \lim _{n} f\left(y_{m}\right) w\left(y_{m}\right)=0 \text { by condition (ii) (b) and (3). }
$$

(4) and condition (ii) (b) imply that $\omega\left(z_{n}\right) \rightarrow 0$ as $n \rightarrow \infty$ for any sequence of distinct points $\left\{z_{n}\right\}$. Consequently, this and (3) give

$$
\lim _{m} \lim _{x_{n}} f\left(y_{m}\right) \omega\left(y_{m}\right)=0 .
$$

Hence $f \in \operatorname{WAP}(G, \omega)$ by ([7], Theorem 6) and Proposition 2.5 .

Thus (ii) implies (i), and our proof is complete.

A mild adjustment of our preceding proof yields the following result, 
on noting that $\Omega(x)$ is finite when either condition holds.

PROPOSITION 3.4. Let $S$ be infinite, discrete and such that $x^{-1}\{y\}$ and $\{y\} x^{-1}$ are finite, for all $x, y \in S$. If $\omega$ is a weight function on $S$, then the following are equivalent :

(i) $\operatorname{WAP}(S, \omega)=C(S, \omega)$

(ii) $S$ is corntable and

$$
\lim _{n} \lim _{m} \frac{\omega\left(y_{m}\right)}{\omega\left(x_{n} y_{m}\right)}=0
$$

for any sequences $\left\{x_{n}\right\}$ and $\left\{y_{m}\right\}$ of distinct points of $S$.

Proof. First we note that (i) implies that ${ }_{x} f \in C(S, \omega)$ whenever $f \in C(S, \omega)$ and $x \in S$, by our definition of $\operatorname{WAP}(S, \omega)$. Now suppose (ii) holds and, on the contrary, $\Omega(x)=\infty$, for some $x \in S$. Then by definition of $\Omega(x)$ (see 2.7 ), there exists a sequence $\left\{y_{m}\right\}$ of distinct points of $S$ such that

$$
\frac{\omega\left(y_{m}\right)}{\omega\left(x y_{m}\right)} \rightarrow \infty \text { as } m \rightarrow \infty \text {. }
$$

since $a^{-1}\{b\},\{b\} a^{-1}$ are finite for all $a, b \in S$, we can find $a$ sequence $\left\{a_{n}\right\} \subset S$ such that $a_{n} x \neq a_{k} x$ whenever $n \neq k$. Let $z_{n}:=a_{n} x$ and note that $\left\{z_{n}\right\}$ is a sequence of distinct points and so

$$
0=\lim _{n} \lim _{m} \frac{\omega\left(y_{m}\right)}{\omega\left(z_{n} y_{m}\right)} \geqslant \lim _{n} \lim _{m} \frac{\omega\left(y_{m}\right)}{\omega\left(a_{n}\right) \omega\left(x y_{m}\right)}=\infty
$$

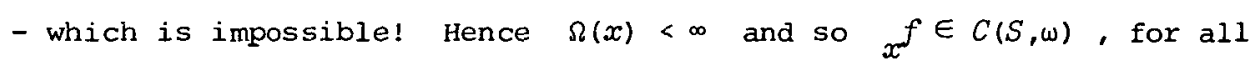
$f \in C(S, \omega)$ and $x \in S$. The rest of the proof can now be modelled on that of Theorem 3.3 .

The analogue of Theorem 3.3 for weighted almost periodic functions yields the following stronger result.

THEOREM 3.5. Let $\omega$ be a continuous weight function on a locally compact topological group $G$. Then the following are equivalent:

(i) $A P(G, w)=C(G, w)$

(ii) $G$ is compact. 
Proof. Suppose (i) holds. By Theorem $3.3 G$ is compact or discrete. If $G$ is discrete then $V_{k}:=\{x \in G: \omega(x)<k\}$ is finite, for all $k \in N$. Since $\left\|f_{\omega}:=\right\| f$. $\|_{G}<\infty$, we must have $f \in C_{o}(G)$ for all $f \in C(G, \omega)$

By Proposition 2.2 we have $w \geqslant 1$ and so

$$
\|f\|_{\omega} \geqslant\|f\|_{G} \text {. }
$$

The map $f \rightarrow f$ of $C(G, w)$ into $C_{0}(G)$ is thus (norm) continuous. Hence $A P(G, \omega) \subseteq A P(G)$ and $A P(G)$ contains a non-zero element of $C_{0}(G)$. The latter implies that $G$ is compact. Thus (i) implies (ii).

By a standard argument the reader can easily verify that (ii) implies (i).

We recall that multiplication in a Banach algebra is said to be regular if the two Arens products on its bidual coincide. If they do not coincide, we have irregular multiplication. For a weighted semigroup algebra $\ell^{l}(S, \omega)$, Craw and Young (see for example [2] or Theorem 1.2 in Chapter 6 of [3]) abtained the following partial characterization of regular multiplication.

PROPOSITION 3.6. Let $\omega$ be a weight function on a cancellative semigroup $S$. Then the following are equivalent:

(i) $\ell^{1}(S, w)$ has irregular multiplication

(ii) there exist sequences $\left\{x_{n}\right\}$ and $\left\{y_{m}\right\}$ of distinct points of $S$ such that the double sequence $\left\{\frac{\omega\left(x_{n} y_{m}\right)}{\omega\left(x_{n}\right) \omega\left(y_{m}\right)}\right\}_{n, m}$ has a non-zero repeated Zimit.

The phenomena of weak almost periodicity of all functions in $C(S, \omega)$ and regularity of multiplication in $\ell^{1}(S, \omega)$ are independent, even for discrete cancellative $S$. This is the message of our next theorem.

THEOREM 3.7. Let $S$ be a free semigroup generated by an infinite countable set, endowed with the discrete topology. Then

(i) $S$ admits a weight function w such that $\operatorname{WAP}(S, \omega)=\mathrm{c}(\mathrm{S}, \omega)$ 
but $\ell^{l}(S, \omega)$ has irregular multiplication

(ii) $S$ admits a weight function $\rho$ such that $\operatorname{WAP}(S, \rho) \neq C(S, \rho)$ but $\ell^{l}(S, 0)$ has regular multiplication.

Proof. (i) Let $D:=\left\{d_{k}: k \in \mathbb{N}\right\}$ be an enumeration of a free generating set for $S$. Every point $x$ in $S$ can be uniquely expressed in the form $x=d_{k_{1}} \ldots d_{k_{p}}$ and we define

$$
\omega(x):=\exp \left(k_{1}+\ldots+k_{p}\right)
$$

Clearly $\omega(x y)=\omega(x) \omega(y) \quad(x, y \in S)$ and $\omega\left(x_{n}\right) \rightarrow \infty$ for any sequence $\left\{x_{n}\right\}$ of distinct points of $S$. In particular, if $\left\{y_{m}\right\}$ is another sequence of distinct points of $S$, then

$$
\lim _{n} \lim _{m} \frac{\omega\left(y_{m}\right)}{\omega\left(x_{n} y_{m}\right)}=\lim _{n \rightarrow \infty} \frac{1}{\omega\left(x_{n}\right)}=0
$$

and

$$
\frac{\omega\left(x_{n} y_{m}\right)}{\omega\left(x_{n}\right) \omega\left(y_{m}\right)}=1 \text {. }
$$

From (5) and Proposition 3.4, we have that $W A P(S, \omega)=C(S, \omega)$; while from (6) and Proposition 3.6 , we have that multiplication in $\ell^{1}(S, \omega)$ is irregular.

(ii) From ([2], page 353), $S$ admits a weight function $\rho$ such that $\ell^{1}(S, \rho)$ has regular multiplication, $\rho\left(x_{n}\right) \rightarrow \infty$ as $n \rightarrow \infty$ for any sequence of distinct points of $S$, and $\rho(x y)=\rho(x)+\rho(y)-I$ for for all $x, y \in S$. So if $\left\{y_{m}\right\}$ is another sequence of distinct points of $S$, we get

$$
\lim _{n} \lim _{m} \frac{\rho\left(y_{m}\right)}{\rho\left(x_{n} y_{m}\right)}=\lim _{n} \lim _{m} \frac{\rho\left(y_{m}\right)}{\rho\left(x_{n}\right)+\rho\left(y_{m}\right)-1}=1
$$

From (7) and Proposition 3.4 , it follows that $W A P(S, \rho) \neq C(S, \rho)$. Let $\omega$ be a continuous weight function on a locally compact topological group $G$. In [6], the author raised the following two questions: Is every right compact element of $L^{I}(G, \omega)$ a left compact 
element? Does the existence of a non-xero right compact element imply that all elements of $L^{1}(G, \omega)$ are right compact? Affirmative answers to both questions follow trivially (or from standard results) as a consequence of the following extension of the main result of [5] and [6].

THEOREM 3.8. Let $\omega$ be a continuous weight function on a locally compact topological group $G$. Then the following are equivalent:

(i) there exists a (non-zero) weakly compact right multiplier of $L^{1}(G, \omega)$;

(ii) $G$ is compact.

Proof. Suppose (i) holds. Then ([6], Theorem 3.1) says that the function

$$
x \rightarrow f(x):=\int \frac{\omega(x y)}{\omega(x)}|g(y)| d \lambda(y)
$$

defined on $G$, for some non-zero $g \in L^{1}(G, \omega)$, vanishes at infinity; where $\lambda$ denotes Haar measure. But

$$
\frac{\omega(x y)}{\omega(x)}=\frac{\omega(x y)}{\omega\left(x y y^{-1}\right)} \geqslant \frac{\omega(x y)}{\omega(x y) \omega\left(y^{-1}\right)}=\omega\left(y^{-1}\right)^{-1}>0 \quad(y \in G)
$$

and so

$$
f(x) \geqslant \int \omega\left(y^{-1}\right)^{-1}|g(y)| d \lambda(y)>0 \quad(x \in G)
$$

Hence $f(x)$ vanishing at infinity can only happen when $G$ is compact. That (ii) implies (i) is evident (see for example [6]).

REMARKS. In fact the conclusion of Theorem 3.8 holds more generally for the case where $\omega$ is any measurable weight function, on noting that with the assistance of Proposition 2.1 one can similarly obtain the result ([6], Theorem 3.1) for such $\omega$.

For a comprehensive study of convolution measure algebras on topological semigroups, we refer the interested reader to our book [3].

\section{References}

[1] R.B. Burckel, Heakly almost periodic flonctions on semigroups. (Gordon and Breach, New York, 1970). 
[2] I.G. Craw and N.J. Young, Regularity of multiplication in weighted group and semigroup algebras. Quart. J. Math. Oxford Ser (2) (1974), 351-358.

[3] H.A.M. Dzinotyiweyi, The analogue of the group algebra for topological semigroups. Qitman Advanced Publishing Program, Boston, London, Melbourne, 1984).

[4] H.A.M. Dzinotyiweyi, Nonseparability of quotient spaces of function algebras on topological semigroups. Trans. Amer. Math. Soc. $272(1982), 223-235$.

[5] F. Ghahramani, Compact elements of weighted group algebras. Pac. J. Math. (to appear).

[6] F. Ghahramani, Weighted group algebra as an ideal in its second dual space. Proc. Amer. Math. Soc. 90 (1984), 71-76.

[7] A. Grothendieck, Critères de compacité dans les espaces fonctionnels genéraux. Amer. J. Math. 75 (1952), 168-186.

[8] N.J. Young, Semigroup algebras having regular multiplication. Studia Math. 47 (1973), 191-196.

Department of Mathematics

University of Zimbabwe

P.O. Box MP 167, Mount pleasant

Harare, Zimbabwe. 